\title{
MITOSIS DETECTION OF HEMATOPOIETIC STEM CELL POPULATIONS IN TIME-LAPSE PHASE-CONTRAST MICROSCOPY IMAGES
}

\author{
Seungil Huh ${ }^{1}$, Sungeun Eom ${ }^{1}$, Dai Fei Elmer Ker ${ }^{2}$, Lee Weiss ${ }^{1}$, and Takeo Kanade ${ }^{1}$ \\ ${ }^{1}$ Robotics Institute, ${ }^{2}$ Department of Biological Sciences and Biomedical Engineering, \\ Carnegie Mellon University
}

\begin{abstract}
Understanding the heterogeneous behavior of hematopoietic stem cells (HSCs) is required for the expansion of the cells without loss of their regenerative capacity. As such, it is essential to establish their lineage relationships by tracking the history of individual cells in a cell population. However, the quality of lineage relationships is often degraded because of undetected or misdetected mitotic events, which lead to missed or inaccurate mother-daughter cell relationships. In this paper, we present an automated mitosis detection method for HSCs in time-lapse phase-contrast microscopy images. Since HSCs are nonadherent, i.e., free-floating in the culture medium, the method is distinguished from the recent mitosis detection methods developed for adherent cells that are attached to the surface of a petri dish. The proposed mitosis detection method detects individual cells in each image frame and subsequently tracks them over time and in so doing identifies newly appeared cells, each of which is considered as a candidate of a newborn cell. Each candidate is then examined to determine whether it is indeed a newborn cell based on temporal change of cell sizes of potential mother and daughter cells. Our method was quantitatively evaluated with 14 HSC populations, each of which is observed for four days, resulting in a precision of $97.4 \%$ and a recall of $96.6 \%$.
\end{abstract}

Index Terms - hematopoietic stem cells (HSC), mitosis detection, cell tracking, probabilistic temporal modeling, microscopy time-lapse image analysis

\section{INTRODUCTION}

Hematopoietic stem cells (HSCs) are blood-forming stem cells and give rise to all blood cell types in the body for cell replacement therapy. In the last decades, the regenerative capacity of HSCs has been utilized to treat bone marrow failure as well as rescue blood function for hematopoietic disorders (e.g., sickle cell anemia, leukemia, lymphoma, immune deficiency disorders and autoimmune diseases) by transplantation of HSCs into patients [1, 2]. However, HSC therapy is limited by the low cell dose available for transplantation as well as the lack of knowledge on how to expand HSCs ex vivo without loss of its regenerative capacity $[3,4]$.
As such, it is essential to establish the lineage relationships between HSCs and their progeny within a population of HSCs. The lineage relationships can provide information on how mechanisms controlling self-renewal and proliferation can be altered in response to the addition of exogenous biochemical signals during in vitro culture to facilitate the study and ex vivo expansion of HSCs. To construct accurate lineage trees, mitosis detection is important since it provides information on mother-daughter relationships. In fact, undetected or misdetected mitosis is a major cause of failure in automated stem cell tracking systems [5].

Recent work has shown great success at detecting mitosis in time-lapse phase-contrast microscopy images; for the review of previous work, we refer to [6]. The most recent work [7] shows that mitosis detection can be automated even under high cell confluence where cells are in contact with one another. This method, however, relies on dramatic morphological and brightness changes of mitotic cells, which happen to adherent cells that are attached to the surface of a petri dish. Therefore, the existing methods may not be applicable to HSCs, which are nonadherent, i.e., free-floating in the culture medium. Due to its nonadherence, such dramatic alterations in cell shape and intensity do not accompany HSC mitosis.

In this paper, we present an automated mitosis method in time-lapse phase-contrast images developed particularly for HSCs. We adopt a recent framework for mitosis detection [6, 7], which comprises detection of spatio-temporal regions for mitosis candidates and validation of the candidates using a temporal probabilistic model. Contrary to the previous work that examines cell appearance and intensity changes, mitosis candidate detection is performed based on cell region detection and tracking, which is fairly tractable in an HSC population due to their limited variation in cell shape and size. For each newly appeared cell during cell tracking, we examine it with its potential mother and sibling cells to determine whether it is a newborn cell as a result of mitosis. For this examination, we present a temporal probabilistic model that models cell size change over time around mitosis. We performed experiments on 14 HSC populations, each of which was captured for four days, to show the effectiveness of our method. 

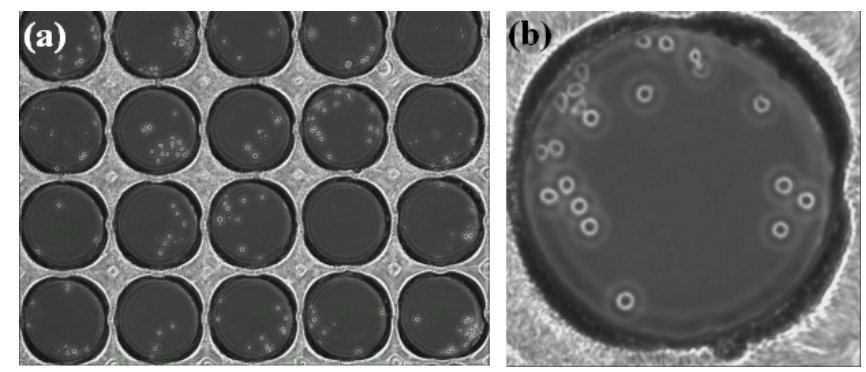

Fig. 1. HSC populations (a) in a microwell array and (b) in a single microwell, imaged with phase contrast microscopy.

\section{METHODS}

In this section, we propose a three-step mitosis detection method: (1) cell region detection and tracking, (2) candidate detection and feature extraction, and (3) candidate validation.

\subsection{Cell Region Detection and Tracking}

We detect cell regions in each image and link them over consecutive frames using a tracking method based on frame-byframe data association.

\subsubsection{Cell Region Detection}

HSCs generally appear as round objects surrounded by bright halos in phase-contrast microscopy images as shown in Figure 1 . Hence, the method based on a template matching with ring filters [8] is effective for the detection of HSCs and is known to outperform other methods [8]. The brief summary of the method is as follows.

Ring filters with different radii are convolved with a given image. In order to prevent the background regions surrounded by cells from being detected, the roundness of ring pattern is computed and multiplied to the convolved result. After thresholding the weighted filter outputs, local maxima are found among them in the space dimension for each radius. Each local peak is then adjusted with a fitting scheme using a quadratic polynomial surface model. For each adjusted local peak, which is considered the center of a cell, the radius with the maximum filtered output in the radius space is selected as the radius of the cell. Fig. 2 shows a sample input image and the detected cell regions on the image.

\subsubsection{Cell Tracking}

Cell regions detected on each image are linked over consecutive frames based on a typical one-to-one frame-by-frame data association method. Specifically, in order to associate a set of tracks up to frame $t-1$, denoted by $\left\{T_{i}\right\}$, with a set of cells at frame $t$, denoted by $\left\{C_{j}\right\}$, the likelihood that each track is associated with each cell is computed based on the distance between them as follows:
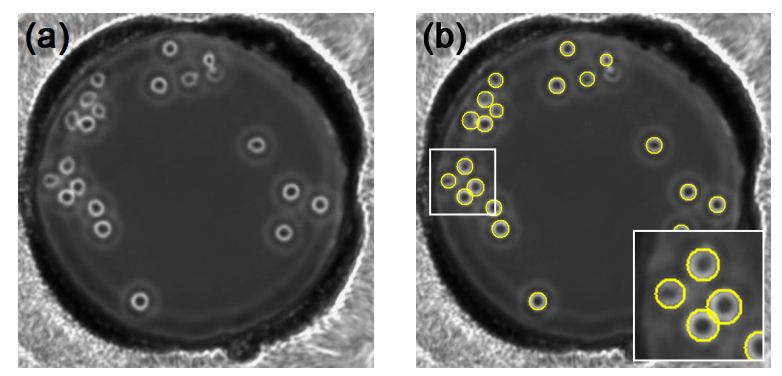

Fig. 2. (a) Sample input image. (b) Cell regions detected on the sample image.

$$
\ell\left(T_{i}, C_{j}\right)=e^{-a \cdot p_{i}} \max \left(1-\left(\frac{\operatorname{dist}\left(T_{i}, C_{j}\right)}{d_{\max }}\right)^{2}, 0\right)
$$

where $a$ is a constant, $p_{i}$ is the number of frames for which $T_{i}$ is unassigned to a cell in a row until reaching frame $t-1$, and $d_{\max }$ is the maximum distance that a cell can move between two successive frames. $e^{-a \cdot p_{i}}$ is multiplied to penalize the tracks that fail to be associated with a cell. Morphological similarity is not taken into account for the likelihood computation because it is not informative due to the limited variation in shape and size among HSCs.

We find the best set of one-to-one correspondences between tracks $\left\{T_{i}\right\}$ and cells $\left\{C_{j}\right\}$ that maximizes the total likelihood using a typical global nearest neighbor (GNN) scheme based on the Munkres algorithm [9]. After the association, there may be several tracks and cells that are not associated with any cell and track, respectively. Such remaining tracks undergo another round of association between tracks and cells in the following frame with the penalty $e^{-a \cdot p_{i}}$ in Eq. (1). If a track is not associated with any cell for $K$ frames in a row, the track is determined to be lost and thus no longer considered. Remaining cells are tracked and observed for $K$ following frames. If a remaining cell is detected for at least $\lceil 0.5 K\rceil$ frames out of the $K$ frames, it is considered a newly appeared cell and a new track initiates from the cell. Otherwise, the remaining cell is regarded as misdetected and thus ignored.

\subsection{Candidates Detection and Feature Extraction}

Among newly appeared cells, we select candidate newborn cells and locate their potential sibling and mother cells. From each of candidates, we extract information on cell size change over time around mitosis.

\subsubsection{Candidate Detection}

A newly appeared cell, denoted by $C_{\text {extra }}$, is considered a candidate newborn cell only if it satisfies two criteria; (1) $C_{\text {extra }}$ is in contact with another cell; (2) the potential mother 


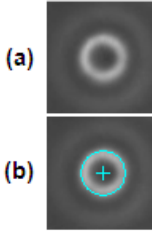

$t-6$

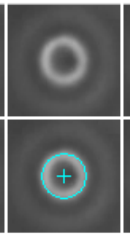

$t-4$

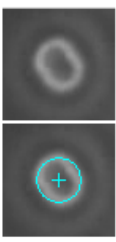

t-2

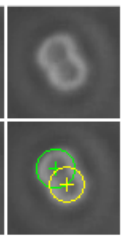

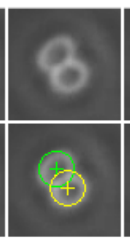

$t+2$

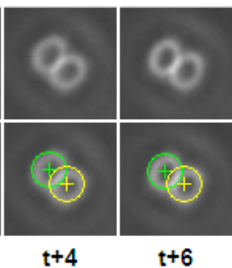

(c)
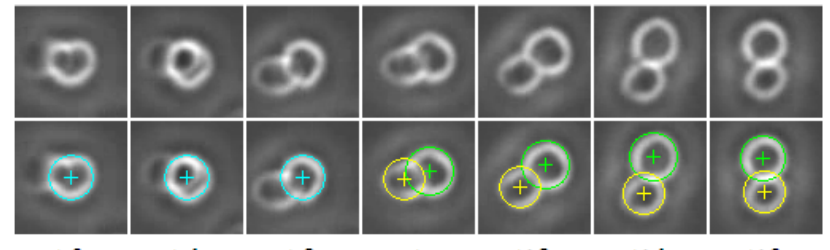

$t-6$

$\mathrm{t}-4$

$\mathrm{t}$

$\mathrm{t}+2$

$t+4$

$t+6$

Fig. 3. (a,c) Patch sequences containing mitosis. (b,d) Detection results of a candidate newborn cell (yellow), and its potential sibling (green) and mother (cyan) cells on (a) and (c), respectively. A circle and a cross in it indicate the boundary and the center of a cell detected, respectively. Newly appeared cells are first detected at frame $t$. (Best viewed in color.)

cell of $C_{\text {extra }}$ is sufficiently greater than $C_{\text {extra }}$ and its potential sibling cell. Note that, during the cell cycle, newborn daughter cells are smaller in size than the mother cell and eventually grow and reach a similar size to the mother cell. The detailed process to locate mitosis candidates is as follows.

Suppose that a newly appeared cell $\left(C_{\text {extra }}\right)$ is first detected at frame $t$. We find the cells in contact with $C_{\text {extra }}$ at frame $t$ as candidate sibling cells. If there is no such a cell, the first criterion is violated and thus $C_{\text {extra }}$ is not further considered. For each of the possible sibling cells $\left(C_{\text {sibling }}\right)$, we find a cell that is associated with $C_{\text {sibling }}$ at frame $t-1$ and regard the cell as the potential mother cell $\left(C_{m o t h e r}\right)$ of $C_{\text {extra }}$ and $C_{\text {sibling. }}$. In other words, we set a hypothesis that $C_{\text {mother }}$ at frame $t-1$ divides into $C_{\text {extra }}$ and $C_{\text {sibling }}$ at frame $t$. From the tracking results, we obtain the trajectory of $C_{\text {mother }}$ for $K$ frames prior to frame $t$, and the trajectories of $C_{\text {extra }}$ and $C_{\text {sibling }}$ for $K$ frames at and after frame $t$. If the average radius of $C_{\text {mother }}$ at frames $t-K$ through $t-1$ is not at least $10 \%$ greater than either of the average radii of $C_{\text {extra }}$ and $C_{\text {sibling }}$ at frames $t$ through $t+K-1$, the second criterion is not satisfied and thus $C_{\text {extra }}$ is disqualified as a candidate newborn cell. Fig. 3 shows examples of a candidate newborn cell and its potential sibling and mother cells.

\subsubsection{Feature Extraction}

From the tracks of $C_{\text {extra }}, C_{\text {sibling }}$, and $C_{\text {mother }}$, we extract the following properties:

- At each of frames $t-K$ through $t-1$

- the radius of $C_{m o t h e r}\left(r_{1: K}^{m}\right)$

- At each of frames $t$ through $t+K-1$

$$
\text { - the radii of } C_{\text {extra }} \text { and } C_{\text {sibling }}\left(r_{1: K}^{e} \text { and } r_{1: K}^{s}\right)
$$

We set $K$ to be 20 in our experiments because $10 \mathrm{~min}$, which is equivalent to 20 frames in our setting, is sufficient for investigating the change of mother or daughter cells due to mitosis. This parameter should be determined based on the image acquisition interval and mitosis duration.

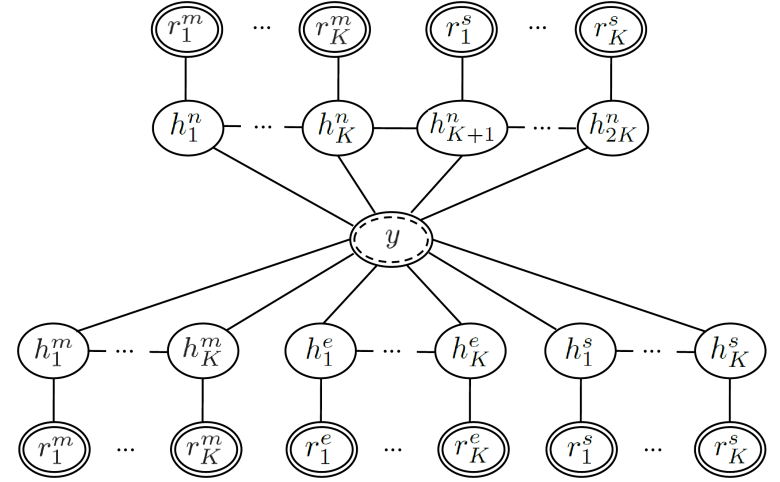

Fig. 4. Graphical representations of the proposed model used for the validation of mitosis candidates. This model combines four Hidden Conditional Random Fields (HCRFs) that model cell size change of a non-mitotic cell, a mother cell, and two daughter cells.

\subsection{Validation of Mitosis Candidates}

By statistically modeling changes in cell size over time during mitosis, we identify true mitosis among the candidates. We formulate a probabilistic model that combines four Hidden CRFs (HCRF) [10] together, as shown in Fig. 4. In this model, binary label variable $y$ indicates the occurrence of mitosis. The first HCRF component with hidden variables $h_{1: 2 K}^{n}$, which are connected with the cell sizes of both candidate mother and sibling cells, models non-mitotic events; if there occurs no mitosis, the candidate mother and sibling cells, which are extracted from the same trajectory, are turned to be one non-mitotic cell and thus the sequentially combined features capture cell size change of a non-mitotic cell over time. On the other hand, the other three HCRF components model cell size change of a mother and two daughter cells separately when mitosis occurs. Combining these four HCRF components together, the proposed model can effectively distinguish mitotic events from non-mitotic ones.

More formally, we define an exponential model for the conditional probability $P(y \mid x, \theta)$, where $x$ is a set of features and $\theta$ is a set of parameters, using the CRF formulation as follows: 


$$
\begin{aligned}
& P(y \mid \mathbf{x}, \theta)=\sum_{\mathbf{h}} P(y, \mathbf{h} \mid \mathbf{x}, \theta) \\
& =\sum_{\mathbf{h}} \frac{1}{Z} \exp \left(\sum_{j=1}^{K}\left(r_{j}^{m} \theta^{n}\left(h_{j}^{n}\right)+r_{j}^{s} \theta^{n}\left(h_{K+j}^{n}\right)\right)+\sum_{j=1}^{K} r_{j}^{m} \theta^{m}\left(h_{j}^{m}\right)\right. \\
& +\sum_{j=1}^{K} r_{j}^{e} \theta^{e}\left(h_{j}^{e}\right)+\sum_{j=1}^{K} r_{j}^{s} \theta^{s}\left(h_{j}^{s}\right)+\sum_{j=2}^{2 K} \theta^{n n}\left(y, h_{j-1}^{n}, h_{j}^{n}\right) \\
& +\sum_{j=2}^{K} \theta^{m m}\left(y, h_{j-1}^{m}, h_{j}^{m}\right)+\sum_{j=2}^{K} \theta^{e e}\left(y, h_{j-1}^{e}, h_{j}^{e}\right)+\sum_{j=2}^{K} \theta^{s s}\left(y, h_{j-1}^{s}, h_{j}^{s}\right) \\
& \left.+\sum_{j=1}^{2 K} \theta^{n y}\left(y, h_{j}^{n}\right)+\sum_{j=1}^{K} \theta^{m y}\left(y, h_{j}^{m}\right)+\sum_{j=1}^{K} \theta^{e y}\left(y, h_{j}^{e}\right)+\sum_{j=1}^{K} \theta^{s y}\left(y, h_{j}^{s}\right)\right)
\end{aligned}
$$

where $Z$ is a partition function. In this formulation, the state function is defined as the radius of a cell and the transition function is defined as the identity function.

In training phase, we maximize the regularized loglikelihood of the entire training samples $\left\{\left(x_{i}, y_{i}\right)\right\}$ to find the best parameter $\theta$. More formally,

$$
\theta^{*}=\arg \max _{\theta} L(\theta)=\sum_{i=1}^{n} \log P\left(y_{i} \mid \mathbf{x}_{\mathbf{i}}, \theta\right)-\frac{1}{2 \sigma^{2}}\|\theta\|^{2}
$$

where $\sigma$ is the variance of a Gaussian prior.

In testing phase, for each mitosis candidate sequence, we compute $P\left(y \mid x, \theta^{*}\right)$, based on which the occurrence of mitosis $y$ is inferred. Due to the space constraint, we omit the detailed learning and inference processes and refer to the related previous work $[10,6,7]$.

\section{EXPERIMENTS}

\subsection{Image and Ground Truth Acquisition}

HSCs were imaged every 30 seconds over the course of 4 days using a Carl Zeiss Axiovert 200M equipped with a ECPlan Neofluar 5X phase objective (Carl Zeiss Microimaging, Thornwood, NY), resulting in 11520 images. Each image contains $1388 \times 1040$ pixels, capturing 14 HSC populations in $3 \times 5$ array of microwells as no cell was seeded in one of the wells.

We manually annotated the time and location that mitosis is completed. More specifically, when the boundary between two newborn cells clearly appear, we marked the contacting point of the two cells, observing 117 mitotic events in total.

\subsection{Results}

We consider a detection a true positive if a contacting point of two newborn cells is detected within spatially 5 pixels and temporally 10 frames ( $5 \mathrm{~min}$ ) from a manually annotated mitosis. If the same mitosis is detected more than once, only one detection is considered true positive, the others false positives.
In the candidate detection step, 234 mitosis candidates were detected. Among the 117 mitosis annotated, $114 \mathrm{mi}-$ tosis were captured by the candidates but the other 3 mitosis were missed. One mitosis was not captured because the mitotic cell overlaps with another cell during the mitosis process. The other two were detected, but temporally beyond the threshold (10 frames), and thus considered missed. As the newborn cells stick to the boundary of the well, they do not show bright and circular appearance; as a result, they were not detected as a cell when they are born, but more than ten frames later.

In the candidate validation step, 116 candidates were determined to be mitosis. Among them, 113 cases were true positives. Therefore, overall $97.4 \%$ precision and $96.6 \%$ recall were achieved for mitosis detection. F-measure and AUC (Area under PR-curve) were $97.0 \%$ and $98.9 \%$. PR-curve was plotted by varying the decision probability for the conditional probability $P(y \mid x, \theta)$, which is originally 0.5 , from 0 to 1 .

\section{CONCLUSION AND FUTURE WORK}

We have proposed a mitosis detection method for HSCs, which are nonadherent, for which previous methods may not be applicable due to the lack of obvious visual cues during HSC mitosis. Our method based on candidate detection with cell tracking and candidate validation with a probabilistic model achieved $97.4 \%$ precision and $96.6 \%$ recall on 14 cell populations.

The proposed method has an advantage over previous methods in that mother-daughter relationships are explicitly revealed so that it can be easily incorporated to cell tracking systems. We leave the empirical analysis as future work.

\section{REFERENCES}

[1] R. Möhle and L. Kanz, "Hematopoietic growth factors for hematopoietic stem cell mobilization and expansion," Seminars in Hematology, vol. 44, no. 3, pp. 193-202, 2007.

[2] I. Martin et al., "A survey on cellular and engineered tissue therapies in europe in 2008," Tissue Eng. Part A, vol. 16, no. 8, pp. 2419-2427, 2010.

[3] S.S. Kelly et al., "Ex vivo expansion of cord blood," Bone Marrow Transplantation, vol. 44, no. 10, pp. 673-681, 2009.

[4] J. Renströma, M. Krgera, C. Peschela, and R.A.J. Oostendorp, "How the niche regulates hematopoietic stem cells," Chemico-Biological Interactions, vol. 184, no. 1-2, pp. 7-15, 2010.

[5] S. Huh et al., "Mitosis detection for stem cell tracking in phase-contrast microscopy images," in Proc. IEEE ISBI, 2011, pp. 2121-2127.

[6] S. Huh et al., "Automated mitosis detection of stem cell populations in phase-contrast microscopy images," IEEE Trans. Med. Imaging, vol. 30, no. 3, pp. 586-596, 2011.

[7] S. Huh and M. Chen, "Detection of mitosis within a stem cell population of high cell confluence in phase-contrast microscopy images," in Proc. IEEE CVPR, 2011, pp. 1033-1040.

[8] S. Eom et al., "Detection of hematopoietic stem cells in microscopy images using a bank of ring filters," in Proc. IEEE ISBI, 2010, pp. 137-140.

[9] F. Bourgeois and J. Lassalle, "An extension of the munkres algorithm for the assignment problem to rectangular matrices," Communications of the ACM, vol. 14, no. 12, pp. 802-806, 1971.

[10] A. Quattoni et al., "Hidden conditional random fields," IEEE Trans. Pattern Anal. Mach. Intell., vol. 29, no. 10, pp. 1848-1853, 2007. 